\section{PWE-113 COELIAC SEROLOGY AT A LARGE DISTRICT GENERAL HOSPITAL, RESULTS IN 6394 PATIENTS}

doi:10.1136/gutjnl-2012-302514d.113

${ }^{1} \mathrm{~K}$ Kodjabashia, ${ }^{1} \mathrm{~S}$ A Haider, ${ }^{2} \mathrm{C}$ Crossland, ${ }^{3} \mathrm{~V}$ Lashmar, ${ }^{1} \mathrm{~S}$ D McLaughlin. ${ }^{1}$ Department of Gastroenterology, Royal Bournemouth Hospital, Bournemouth, UK; ${ }^{2}$ Department of Histopathology, Royal Bournemouth Hospital, Bournemouth, UK; ${ }^{3}$ University of Southampton, Southampton, UK

Introduction Coeliac disease has an estimated UK prevalence of $1 \%$ and is an important, common cause of many gastro-intestinal and non-gastro-intestinal symptoms. Coeliac serological blood tests are commonly performed in both primary and secondary care. Positive coeliac serology occurs in patients with: (i) Coeliac disease, (ii) Latent Coeliac disease, (iii) Dermatitis herpetiformis. The UK national institute for health and clinical excellence (NICE), ${ }^{1}$ British society of gastroenterology guidelines (BSG) and American Gastroenterology Association (AGA) ${ }^{2}$ guidelines recommend that all patients with positive coeliac serology undergo duodenal biopsy since diagnosis of coeliac disease requires both positive serology and typical histological findings.

Methods We reviewed the results of all coeliac serology tests performed at our hospital laboratory in the previous 12 months. The case notes for all patients with positive results were reviewed.

Results 6394 endomysial antibody results were performed on adult patients between 1 October 2010 and 30 September 2011. 100 $(1.6 \%)$ were positive. Of these $67(67.0 \%)$ underwent biopsy. 50 (74.6\%) had histological evidence of coeliac disease; $5(7.5 \%)$ were inconclusive and $11(16.4 \%)$ had no evidence of coeliac disease. Of those who did not undergo biopsy $11(33.0 \%)$ were known to have $\mathrm{CD}$ or refused D2 biopsy. In $22(21.6 \%)$ patients gastroenterological follow-up had not been arranged, of these $16(72.7 \%)$ tests had been arranged in primary care.

Conclusion In this study $1.6 \%$ of those tested had serology suggestive of coeliac disease, this is marginally larger than expected by chance and suggests that testing was not appropriately targeted. Surprisingly $21.6 \%$ of positive tests did not have appropriate followup arranged. We suspect these findings are not confined to our institution. Our findings suggest that engagement and education of non-gastroenterology colleagues, particularly those in primary care is important in order that patients receive appropriate treatment and conform to AGA, BSG and NICE guidelines. We plan in future that all positive coeliac serology test reports be issued with the advice that referral to a gastroenterologist is recommended.

Competing interests None declared.

\section{REFERENCES}

1. AG1. Recognition and Assessment of Coeliac Disease, Clinical Guidelines, CG 86 Issued: May 2009

2. AGA Institute. AGA Institute medical position statement on the diagnosis and management of coeliac disease. Gastroenterology 2006;131:1997-80.

\section{PWE-114 DOES GASTROINTESTINAL INFECTION TRIGGER COELIAC DISEASE?}

doi:10.1136/gutjnl-2012-302514d.114

${ }^{1} \mathrm{~K}$ Evans, ${ }^{* 1} \mathrm{~S}$ M Barrett, ${ }^{1,2} \mathrm{~A}$ Smythe, ${ }^{1} \mathrm{D}$ S Sanders, ${ }^{1} \mathrm{~A}$ Arnout. ${ }^{1}$ Sheffield Teaching Hospitals NHS Trust, Sheffield, UK; ${ }^{2}$ University of Sheffield, Sheffield, UK

Introduction It is thought that a "second hit" is required to trigger coeliac disease in genetically susceptible individuals. Various infective agents have been postulated as the second hit but there is little evidence to support this. We aimed to establish the recall rate of antecedent gastrointestinal infection in patients with coeliac disease, and the prevalence of undetected coeliac disease in those with stool culture proven gastroenteritis.
Methods Group A comprised histologically proven patients with coeliac disease ( $n=233,61$ male, median 60 years) who were asked to complete a validated questionnaire and then compared to healthy controls ( $\mathrm{n}=219,79$ male, median 46 years), and controls with inflammatory bowel disease (IBD) $(n=196,124$ males, median 56 years). Group B were patients with stool culture proven gastroenteritis ( $n=101,48$ males, median 57 years) who underwent serologic testing for coeliac disease (endomysial antibody [EMA], tissue transglutaminase [tTG], immunoglobulin A [IgA]). They were compared with healthy controls ( $n=1200,447$ male, median 46 years). Those with positive serology underwent endoscopy and duodenal biopsy.

Results In Group A 69/233 (29.6\%) with coeliac disease, and 53/196 $(27.1 \%)$ with IBD reported having a gastrointestinal infection within the 12 months prior to diagnosis. In both diseases this was significantly greater than in healthy controls 15/219 (6.8\%) $(\mathrm{p}<0.0001)$. In Group B 94/101 (93\%) were coeliac antibody negative. The demographics, serology and biopsy results of the seven stool-culture positive subjects with positive coeliac serology are shown in Abstract PWE-114 table 1. The prevalence of coeliac disease in patients with stool culture positive gastroenteritis was $2.97 \%$. This was higher than in healthy controls $(12 / 1200,1 \%)$ $(p=0.10)$. In Group B the gastroenteritis pathogen was identified as Campylobacter species in 96/101 (95.0\%), Salmonella species in 4/ 101 (4.0\%), and Shigella in 1/101 (1.0\%). One participant had IgA deficiency. This individual had normal IgG titres, IgG EMA and IgG tTG

Abstract PWE-114 Table

\begin{tabular}{lllllrl}
\hline Case number & Age & Sex & Pathogen & EMA & tTG & Duodenal biopsy \\
\hline 1 & 70 & M & Campylobacter & 0 & 23 & Marsh 3c \\
2 & 49 & M & Campylobacter & 0 & 19 & Marsh 0 \\
3 & 34 & M & Campylobacter & 0 & 94 & Marsh 0 \\
4 & 78 & M & Campylobacter & 1 & 300 & Marsh 3c \\
5 & 48 & M & Campylobacter & 0 & 56 & Marsh 0 \\
6 & 44 & M & Campylobacter & 1 & 300 & Marsh 3a \\
7 & 32 & F & Campylobacter & 0 & 169 & Marsh 1 \\
\hline
\end{tabular}

Conclusion Patients with coeliac disease have a recall rate of previous gastrointestinal infection similar to those with inflammatory bowel disease, and significantly greater than healthy controls. In coeliac disease gastrointestinal infection may well be the "second hit" required to trigger disease but further work is required.

Competing interests None declared.

\section{PWE-115 A 13-YEAR, SINGLE-CENTRE EXPERIENCE OF GLUCOSE HYDROGEN BREATH TESTING}

doi:10.1136/gutjnl-2012-302514d.115

K Evans, ${ }^{*}$ E Lunn, S Raza, D Elphick, D S Sanders. Sheffield Teaching Hospitals NHS Trust, Sheffield, UK

Introduction Historically, the gold standard for diagnosing small intestinal bacterial overgrowth (SIBO) has been quantitative culture of jejunal aspirate. However this test is costly and invasive. More commonly in clinical practice the glucose hydrogen breath test (GHBT) is used. We aimed to determine which clinical features and baseline laboratory investigations indicate a high likelihood of SIBO as defined by positive GHBT.

Methods We undertook a retrospective analysis of records for all patients referred for GHBT at a single teaching hospital over a 13year period 1998-2010. Data collected included age, sex, baseline and peak hydrogen levels, previous surgical procedures, 